\title{
LE MOUVEMENT LITTERAIRE AUX ANTILLES ET A LA GUYANE ${ }^{1}$ )
}

PAR

\section{René Maran ${ }^{2}$ )}

Il n'est personne qui ne sache aujourd'hui la passion que les noirs ont pour la musique. On a cru longtemps que l'auteur de l'Essai sur l'Inégalité des Races Humaines avait été le premier à la signaler. Le comte de Gobineau n'a fait que propager de son autorité une opinion que bon nombre d'ouvrages mineurs du xvine siècle avaient déjà publiée. On relève par exemple dans un passage de Saint-Domingue à la veille de la Révolution où le baron de Wimpffen parle d'un orchestre nègre: „Cet orchestre est composé de deux violons bien supérieurs pour le talent qu'exige leur emploi à la plupart de nos racleurs européens. Ils ont encore sur ceux-ci l'avantage de ne pas être les instruments passifs du plaisir des autres, car ils entrent si bien dans le sens de la chose que la partie de leur corps qui est assise frétille dans un accord parfait avec le pied qui bat la mesure et le bras qui conduit l'archet."

Voilà qui est aussi parfaitement observé que rendu. De Gobineau en a tiré la conclusion au chapitre viI du livre second de

1) Dit artikel, evenals dat van Swanzy over de literatuur in de Britse Westindische gebieden in de $32 \mathrm{e}$ jrg. van de W.I.Gids, p. 217, was oorspronkelijk bestemd voor Eldorado. De redactie van dit tijdschrift stond het welwillend aan de W.I.Gids af, toen Eldorado ophield te verschijnen. Het dateert van 1949.

2) RENÉ MARAN, geboren op Martinique, studeerde in Bordeaux, kwam bij het binnenlands bestuur in Frans Oost-Afrika. Met zijn roman Batonala verwierf hij de Prix Goncourt in 1921, doch moest toen de bestuursdienst verlaten.

Auteur van Les pionniers de l'Empire, Le livre de la Brousse en vele artikelen. (Zie Présence Africaine no. 8-9, p. 397 en no. 10-11, p. 210; ook Robert Delavignette "L'accent africain dans les lettres françaises" in La Nef novembre 1945.) 
son fameux ouvrage. "L'élément noir," y déclare-t-il, ,,est indispensable pour développer le génie artistique dans une race, parce que nous avons vu quelle profusion de feu, de flamme, d'étincelles, d'entraînement, d'irréflexion réside dans son essence, et combien l'imagination, ce reflet de la sensualité, et toutes les appétitions de la matière le rendent propre à subir les impressions que subissent les arts, dans un degré tout à fait inconnu aux autres familles humaines. C'est mon point de départ, et s'il n'y avait rien à ajouter, certainement le nègre apparaîtrait comme le poète lyrique, le musicien, le sculpteur par excellence."

Qui parle de musique nègre ne peut pas ne pas toucher mot des danses qu'elle anime. Chez le noir, danse et musique sont inséparables et en quelque sorte consubstantielles. L'une mime, l'autre parle. Toute musique est prétexte à danse, toute danse prétexte à musique en Afrique noire. Il en est de même dans tous les pays que le commerce du bois d'ébène a peuplé de nègres. Le dépaysement et la transplantation de ces danses parviennent à peine à les défigurer. Presque toutes, de quelque nom qu' on les nomme, black bottom ou tango, cake walk ou biguine, rumba ou samba, dérivent du calenda, originaire des côtes de Guinée, et selon toute probabilité du royaume disparu d'Arada.

Ces danses, et la musique qui leur donne vie, n'expriment pas toujours que joie physique. Il leur arrive souvent, au contraire, d'être mélancolie, chagrin, souffrance, douleur, révolte, espoir désespéré, élan de l'âme, mysticisme religieux, plainte étouffée d'une race demandant à la prière de la délivrer du complexe d'infériorité dans laquelle elle se débat plus ou moins depuis des siècles. De là sont nés les ,blues” et les „spirituals," chants poignants jaillis du plus profond de l'être, effusions nourries de cette fervour absolue et de cette foi constructive qui rendent exemplaires les premiers temps de l'Eglise et les plus beaux jours du moyen âge.

En général, danse, musique et poésie parlent le même langage chez la plupart des peuples jeunes et chez la plupart des races qui ne sont pas encore sorties de l'enfance. Danseur-né, musicien-né, le noir est aussi un poète-né. Il est par conséquent naturel que des poètes de couleur tels que Laurence Dunbar, Countée Cullen Claude Mc Kay, Jean Toomer, James Weldon Johnson, Georgia Douglas Johnson, Langston Hughes et Richard Wright soient de nos jours aux Etats-Unis l'honneur de la littérature américaine Et il est non moins naturel que la littérature française contemporaine soit fière, de son côté, de voir son patrimoine s'enrichir 
de recueils publiés par des poètes de couleur ayant vu le jour qui à Haïti, qui à la Guadeloupe, qui à la Martinique, qui à la Guyane, et dont les plus remarquables - Daniel Thaly, Aimé Césaire, Jacques Roumain, Gilbert Gratiant, Emmanuel-Flavia Léopold et L-G. Damas - ont pour émules ou disciples des Etienne Léro, des Guy Tirolien, des Léon Laleau, des Jean-F. Brière, des René Belance et des Paul Niger.

Américains de couleur et Français de couleur se rencontrent en ceci: les premiers se sentent Américains corps et âme, les seconds corps et âme Français.

I, too sing America,

s'écrie Langston Hughes dans un poème dont la célébrité a depuis longtemps franchi l'Atlantique.

I am the darker brother.

They send me to eat in the kitchen

When company comes.

But I laugh,

And eat well;

And grow strong.

To-morrow

I'll sit at the table

When company comes,

Nobody'll dare

Say to me:

- Eat in the kitchen -

Then.

Besides, they'll see how beautiful I am

And be ashamed, -

I, too, am America.

Il n'est pas un mot de cet orgueilleux poème que les écrivains de couleur nés à Haïti et dans les départements français de la Guadeloupe, de la Martinique et de la Guyane ne fassent entièrement leur, après avoir pris soin de remplacer le $I$, too, am America, qui sert désormais de cri de ralliement aux noirs des Etats-Unis, par cet autre cri de ralliement: Nous aussi, nous sommes Français!

Américain ou Français, l'écrivain de couleur est essentiellement lyrique. Il est de même essentiellement surréaliste et existentialiste. Lyrisme, surréalisme et existentialisme coexistaient en lui bien avant qu'on ne songeât à les appeler ainsi. Ces trois modes d'expression continueront à coexister en lui quand ils auront cessé de plaire. Il passe de l'un à l'autre, et du réalisme à l'idéa- 
lisme, avec l'aérienne aisance d'un Homère ou d'un Aristophane. On lui reconnaît chaque jour davantage cette faculté de mutation, à mesure qu'on pénètre mieux les secrets de la race noire, ainsi que la richesse et la variété de ses moeurs, de ses traditions et de son folklore.

Il est par conséquent dans la logique de l'oeuvre de libération totale que la France poursuit depuis des siècles dans l'ensemble des territoires composant aujourd'hui l'Union Française, que les écrivains français de couleur, romanciers, poètes ou essayistes, cédant à la plus noble émulation, aient fini par mêler leurs travaux personnels à ceux de leurs compatriotes et confrères européens, et à marcher avec assurance dans la voie que leur a ouverte Alexandre Dumas père et le poète des Salaziennes et d'Epaves, Auguste Lacaussade, mulâtre réunionnais que Victor Hugo honorait de son amitié, et dont certains poèmes, fort beaux du reste, extraits d'une oeuvre dont l'Académie Française avait distingué le lyrisme en lui décernant l'une de ses récompenses les plus appréciées, le Prix Bordin, ont longtemps figuré dans les recueils de „Morceaux Choisis” en usage, à l'époque 1900, dans la plupart des lycées et collèges des Universités françaises.

L'île où la jeune Françoise d'Aubigné, venue de Niort, a passé, dit-on, quelques mois de sa petite enfance, avant de rentrer définitivement en France, où les jeux du destin devaient lui donner pour mari Paul Scarron, auteur du Roman Comique et du Virgile Travesti, puis faire d'elle la marquise de Maintenon et l'épouse morganatique de Louis XIV, la Marţinique, pour appeler cette île par son nom, est depuis une quinzaine d'années le centre d'un extraordinaire mouvement littéraire qui rayonne sur Haïti, la Guadeloupe et la Guyane Française.

Les porte-flambeaux de ce mouvement littéraire sont: René Jadfard, L-G. Damas et Léo J. Cresson pour la Guyane; Jacques Roumain, Léon Laleau et Jean-F. Brière pour Haïti; Jean-Louis Baghiv'o, Guy Tirolien et Paul Niger pour la Guadeloupe; enfin, pour la Martinique: Aimé Césaire, Claude et Magdeleine Carbet, Mayotte Capécia, Henry Maurice, qui écrit sous le pseudonyme aussi puéril que mauvais de Petit-Mau, René Clarac, Joseph Zobel et Raphaël Tardon.

Un mot d'abord de quatre des principaux romanciers qu'il a révélés: Jacques Roumain, Joseph Zobel, Mayotte Capécia et Raphaël Tardon.

Le haïtien Jacques Roumain est le poète de Bois d'Ebène et 
le romancier de Gouverneurs de la Rosée. Ce titre surprend par son étrangeté. Jacques Roumain, que la mort a malheureusement ravi trop vite aux lettres françaises, s'est chargé de l'expliquer lui même à ses lecteurs. „La terre,” leur dit-il, ,,c'est une bataille jour pour jour, une bataille sans repos: défricher, planter, sarcler, arroser jusqu' à la récolte, et alors tu vois ton champ mûr couché devant toi le matin, sous la rosée, et tu dis: moi Untel, gouverneur de la rosée, et l'orgueil entre dans ton coeur!"

Est-il plus admirable image que celle-ci, dont le poétique hermétisme semble tiré du folklore afro-haîtien? Tous les laboureurs, tous les tâcherons qui peinent par le monde, du matin au soir, dans leurs champs ou leurs plantations, sont des ,gouverneurs de la rosée." La sueur de leurs travaux réclame chaque jour ses bienfaits. C'est grâce à leurs soins que plantes potagères et céréales ploient, du crépuscule à l'aurore, sous le poids du liquide qui les nourrit.

Ce roman du sol et de l'eau se passe en pays noir. Le sang y rachète le sang, la mort y engendre la vie. La révolte humaine contre la fatalité fleure un subtil parfum ,Vieille France”. Parée de surcroît de termes du cru et de proverbes dont l'origine africaine n'est pas niable, cette oeuvre âpre et forte, revendicative et justicière, emprunte tantôt la manière d'un Giono débarrassé de tout procédé littéraire, tantôt celle d'un Péguy qui, né dans une de ces îles paresseuses "ou la nature donne ces arbres singuliers et ces fruits savoureux" chantés par Baudelaire, aurait trié, avant que de s'en servir, le tout-venant des apports du surréalisme. On ne saurait faire à ce roman hors de pair compliment plus beau.

M. Joseph Zobel, en ce qui le concerne, est loin de valoir Jacques Roumain. Son mérite, pour être moindre, est malgré tout digne d'estime. Il est d'autre part, et cela ne gâte rien, le premier écrivain martiniquais qui ait songé à tirer de sa terre natale un roman strictement régionaliste. On plonge en effet en plein régionalisme dès les premières pages de Diab'-la - tel est le titre de l'ouvrage qui lui a valu, il y a quatre ans, le Grand Prix Littéraire des Antilles - et l'on n'en sort plus. Le portrait de Diab'-là Diab'-là est le nom que porte le héros dans lequel M. Joseph Zobel a personnifié le prolétariat martiniquais - est un portrait qu'on ne peut imaginer nulle part ailleurs. Il respire, si l'on peut du moins s'exprimer ainsi, le pays qui l'a formé et les horizons qui veillent sur lui. „C'était,” au dire de son biographe ,un nègre rouge d'environ trente-cinq ans, avec des cheveux coupés en 
brosse de chiendent sur un front dégagé, et un peu aplati, qui surplombait des yeux larges, pleins d'un blanc jauni, mais qui se resserraient comme des yeux d'oiseau de proie quand ils fixaient. Pour un nègre, son nez était assez régulier, et soufflait une haleine puissante dans la broussaille touffue de sa moustache noire retombant sur la lèvre. L'autre lèvre se montrait sensuellement charnue et rembrunie par un long usage du tabac. Sa mâchoire, très musclée, saillait un peu sous les oreilles. Et sous sa vestekaki,fermée presque sous son menton dur, sa respiration soulevait lourdement sa poitrine bloquée entre les gonflements massifs de ses épaules."

Tout le livre est écrit de la même encre. Les gaucheries y bousculent les incorrections, les lourdeurs les impropriétés, les passages les mieux venus d'absurdes incohérences, les expressions du cru les fautes grammaticales, dans lesquelles peut-être il ne faut voir parfois que des expressions du cru. Mais le tout court d'un tel train, fait un tel feu d'artifice, qu'on en est étouffé, ébloui, au point qu'on oublie souvent tout esprit critique pour se laisser aller à son seul plaisir.

Tout comme M. Joseph Zobel, Mme Mayotre CAPÉcia est née à la Martinique, et, tout comme lui, sous une bonne étoile, son premier roman, Je suis une Martiniquaise, ayant reçu non seulement les louanges de l'ensemble de la critique métropolitaine, mais aussi le Grand Prix Littéraire des Antilles pour l'année 1948.

Son second roman, La Négresse Blanche, sera sans doute favorisé d'un accueil analogue. Ce qui fait toute la valeur de ce nouvel ouvrage, c'est d'abord l'art de conter où excelle son auteur, ensuite sa science innée de mener une intrigue de bout en bout jusqu'à son parfait achèvement, et enfin qu'elle expose en toute impartialité le complexe, délicat et douloureux problème des conflits interraciaux aux Antilles, et, pour peu qu'on le transcende et le généralise, partout où des races différentes sont obligées de cohabiter.

Ce problème, l'un des plus graves du temps présent, Mme Mayotte Capécia, qui est elle-même une femme de couleur, le rend plus émouvant en prêtant à la belle Isaure, son héroïne, les pensées et les réflexes de la plupart des femmes antillaises qui se minent d'avoir plus ou moins de sang noir dans les veines.

Isaure et ses pareilles sont au fond les seules artisanes de leur souffrance. Fières, on ne sait pourquoi, d'un seul des deux sangs qui battent dans leur pouls, le blanc, elles ont honte en revanche, on se demande pourquoi, du second des deux sangs qui les animent: le noir. Elles se fabriquent, ce faisant, un complexe d'infé-

West-Indische Gids XXXIII 
riorité dont elles ne se guériront que le jour où, imposant silence à leur lâcheté et à leur mauvaise conscience, elles réconcilieront en elles les deux races dont elles sont issues et les aimeront d'un même amour.

Le quatrième de ces romanciers antillais, M. RAPHAËL TARDON, a publié, il y a deux ans, Starkenfirst, roman dur et puissant, titulaire, lui aussi, du Grand Prix Littéraire des Antilles, et digne, si les jurys parisiens n'écartaient systématiquement de toute compétition les romans régionalistes d'expression extra-métropolitąine soumis à leur examen, d'un des grands prix littéraires français de fin d'année.

La Caldeira, qu'il a fait récemment paraître, éclate, tout comme Starkenfirst, des dons propres au romancier-né. C'est l'histoire de l'épouvantable éruption qui détruisit de fond en comble la ville de Saint-Pierre-de-la-Martinique en 1902. Mais La Caldeira n'est pas que le roman de l'éruption du Mont Pelé. Elle est aussi le roman des luttes politiques, compliquées de préjugés raciaux qui divisent entre eux les créoles, - ce mot doit être pris dans son acception première, - les mulâtres et les noirs de la Martinique. Il vaut, par conséquent, aussi bien pour les Antilles que pour les pays et les nations où les préjugés raciaux ont cours.

Ce qui fait le tragique de ce roman aux situations parfois shakespeariennes, c'est que ces luttes se donnent carrière, s'exaltent et passent la mesure, pendant que le destin balance le sort de toute une ville. On se hait, de nuance de teint à nuance de teint, et, tout en se haïssant, on danse sur un volcan.

M. Raphaël Tardon fouaille avec autant de vigueur que de gouaille la stupidité de ces passions politico-raciales qui ont obnubilé l'instinct de conservation des Pierrotins jusqu'à la veille de la catastrophe. C'est vraiment du beau et bon travail, et de la plus grande utilité. L'auteur de La Caldeira a eu le courage de dire leur fait à ses compatriotes blancs et noirs. Il peut d'ores et déjà être sûr qu'aucun d'eux ne lui en saura gré.

La Réunion a longtemps été la terre d'élection des poètes d'outre-mer. Les Antilles de langue française, grâce à Aimé Césaire, à Jacques Roumain, à Emmanuel-Flavia Léopold et à Gilbert Gratiant, sont en passe de lui ravir ce renom mérité.

EMMANUEL-FLAVIA LÉOPOLD, âme chantante et nostalgique, nourrit des cadences de ses promenades mentales chacun de ses recueils de poèmes: Suite pour un Visage, Le Vagebond, l'Automne à Septfonds et Adieu foulards, adieu madras. 
Classique, son vers, qui ne porte jamais si loin que lorsqu'il se plie aux plus strictes disciplines de la métrique traditionnelle, est riche des emprunts qu'il a faits au romantisme, à l'école parnassienne, au symbolisme, au vers-librisme, voire même à un surréalisme assagi.

L'éclatant génie de la comtesse de Noailles a marqué ses débuts de l'influence de son verbalisme. Il semble d'autre part qu'il ait subi celle d'Henri de Régnier. On décèle, en tout cas, l'une et l'autre dans l'ode à la Louange du matin, où il s'écrie:

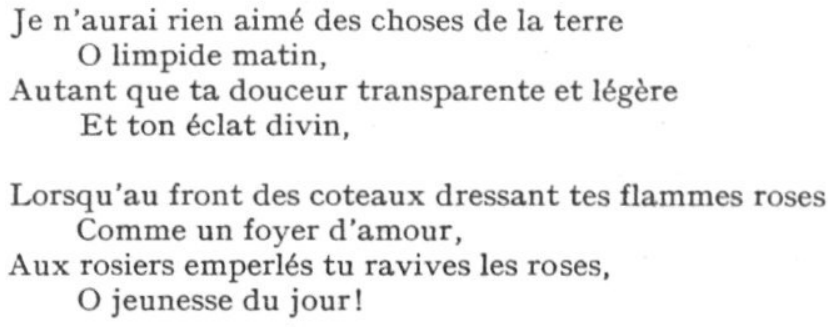

Toute oreille exercée ne peut manquer de reconnaître, dans ces deux strophes glanées au hasard, l'accent de la comtesse de Noailles marié, si l'on peut dire, à la manière de l'auteur de $L a$ Sandale ailée.

M. Emmanuel-Flavia Léopold a finalement cédé, à mesure qu'il prenait peu à peu conscience de sa personnalité profonde, au courant élégiaque dont les eaux l'entraînaient à son coeur défendant. Le poète de Suite pour un Visage est en effet un petit neveu d'André Chénier, à qui rien ne serait étranger de l'oeuvre de Francis Jammes et de Charles Guérin.

Un de ses plus beaux poèmes, peut-être même le plus beau qu'il ait publié jusqu'ici, le montre en pleine possession de son talent, comme le prouvent les quelques vers ci-dessous.

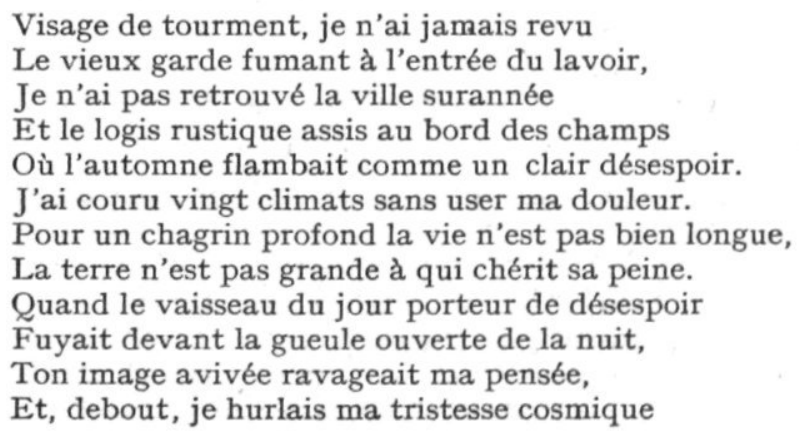


Au Dieu qui se cachait derrière les étoiles.

Mais je n'ai jamais vu tomber parmi les branches

Ton nom que je mourrai sans avoir prononcé.

La résignation de ces deux derniers vers, où l'on retrouve comme la voix de Léon Deubel, ne laissait guère prévoir l'excursion éblouie qu'Emmanuel-Flavia Léopold a faite sur les terres du surréalisme; d'où il est revenu enrichi de Deux Poèmes et de l'Automne à Septfonds, et heureux d'avoir eu un moment pour guides Aimé Césaire et Gilbert Gratiant.

Leur double et fraternel exemple l'a restitué à la Martinique, son pays natal. Il l'a fait revivre dans Adieu foulards, adieu madras, en puisant, pour ce faire, dans les croyances et les récits de son folklore, la substance de ses chants chargés d'émotion et de mélancolie.

M. Emmanuel-Flavia Léopold peut être fier d'avoir écrit Adieu foulards, adieu madras.

De Jacoues Roumain et de Gilbert Gratiant, on peut dire que c'est Aimé Césaire qui les a révélés à eux-mêmes. Le premier est haïtien, le second martiniquais. Le premier est né à Port-auPrince en 1907, le second à Fort-de-France en 1901. Le premier a publié Gouverneurs de la Rosée, roman régionaliste et social de tout premier ordre, ainsi que Bois d'Ebène, titre d'un recueil de poèmes qui a vu le jour en 1945. On doit au second trois ouvrages de poésie: Poèmes en vers faux, An Moué, poésies populaires en patois martiniquais, et Credo des Sangs-Mêlés, son chef-d'oeuvre. Chez tous deux enfin le politique complète l'écrivain. De là vient que les poèmes de ces deux poètes élevés et formés à la française sont des chants de révolte, et que chez l'un comme chez l'autre, comme chez tous les poètes de couleur dont M. Léopold Sédar Senghor a recueilli les morceaux choisis dans son Anthologie de la Nouvelle Poésie Nègre et Malgache de Langue Française et M. L.-G. Damas dans ses Poètes d'Expression française, grondent les mêmes colères revendicatives et les mêmes condamnations vengeresses.

Je sais un silence,

clame Jacques Roumain, dans le prélude de Bois d'Ebène,

un silence de vingt-cinq mille cadavres nègres,

de vingt-cinq mille cadavres de Bois d'Ebène

Sur les rails du Congo-Océan.

Mais je sais

des suaires de silence aux branches des cyprès, 


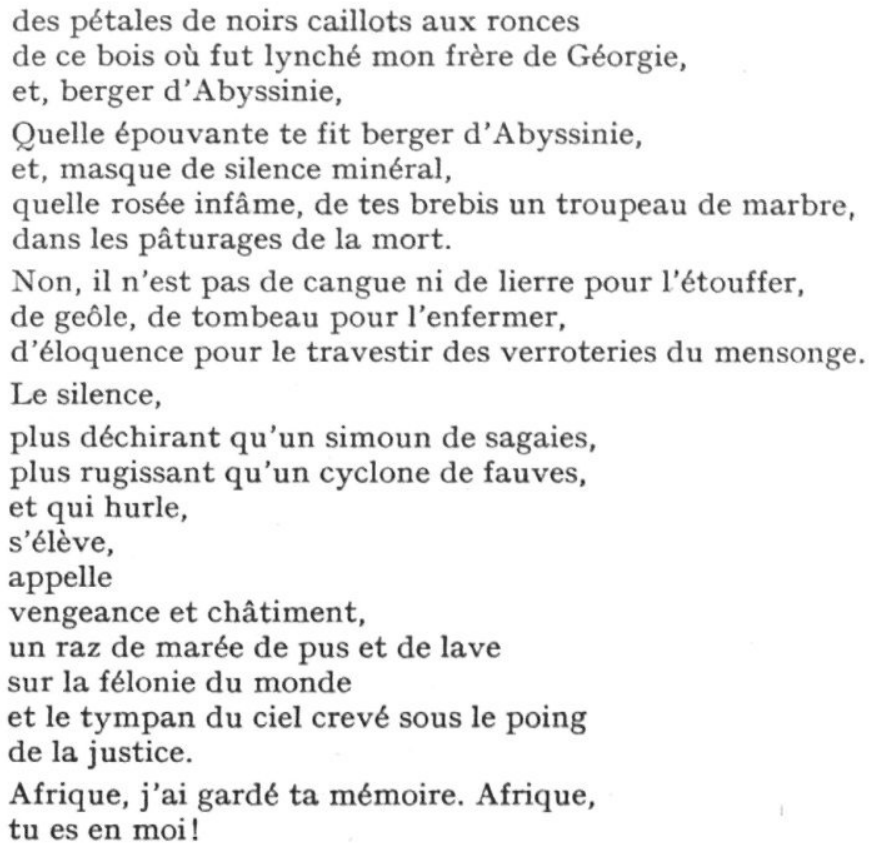

Gilbert Gratiant invective lui aussi, mais en termes plus nuancés, plus modérés, contre les séquelles de la traite des nègres, la haineuse imbécillité des préjugés raciaux et les intolérables méfaits du capitalisme colonialiste.

Quant à AIMÉ CÉSAIRE, de qui l'on dira peut-être un jour qu'il est le Victor Hugo du surréalisme, aucun de ses amis n'ignore qu'il a mis le lyrisme de son délire verbal au service exclusif des revendications raciales et sociales les plus justifiées, par le truchement de son Cahier d'un retour au pays natal et de ses Armes miraculeuses, que le pape de l'Ecole Surréaliste, André Breton, considère, étant orfèvre en la matière, comme des chefs-d'oeuvre.

Il est regrettable que la trop courte citation qui va suivre ne puisse donner qu'un très faible aperçu du souffle qui soulève d'un bout à l'autre l'admirable poème en prose rythmée qu'est Cahier d'un retour au pays natal, et de la densité de la pensée qui l'anime.

Eïa pour ceux qui n'ont jamais rien inventé, pour ceux qui n'ont jamais rien exploré, pour ceux qui n'ont jamais rien dompté!

Eïa pour la joie!

Eïa pour l'amour!

Eïa pour la douleur aux pis de larmes réincarnées! 
Donnez-moi la foi sauvage du sorcier.

Donnez à mes mains puissance de modeler.

Donnez à mon âme la trempe de l'épée.

Je ne me dérobe point. Faites de ma tête une tête de proue,

et de moi-même, mon coeur, ne faites ni un père, ni un frère,

ni un fils, mais le père, mais le frère, mais le fils;

ni un mari, mais l'amant de cet unique peuple.

Faites-moi rebelle à toute vanité, mais docile à son génie,

comme le poing à l'allongée du bras!

Faites-moi commissaire de son sang!

Faites-moi dépositaire de son ressentiment!

Faites de moi un homme de terminaison!

Faites de moi un homme d'initiation!

Faites de moi un homme de recueillement!

Mais faites aussi de moi un homme d'ensemencement!

Faites de moi l'exécuteur de ces hautes oeuvres!

Mais, les faisant, mon coeur, préservez-moi de toute haine!

On pourrait reproduire nombre de morceaux plus violents et plus significatifs. Les uns sont de longues plaintes, les autres des sortes d'ïambes en prose. Satires violentes débordant d'exaspération ou voceri désespérés, tous véhiculent une souffrance, charrient une douleur provenant du fond des âges, douleur et souffrance d'une race que l'Europe chrétienne a déportée aux Etats-Unis, dans les îles de la mer Caraïbe, en Amérique Latine, à la Réunion et à Madagascar pour des fins en voie de disparition à l'heure actuelle.

De là vient que tous sont plus ou moins en proie à ce rythme secret, à cet égarement vaticinateur et sacré qui caractérisent le génie de la race noire. Ce rythme, cet égarement, l'exultation de leur exaltation, leur humour singulier, leur tristesse, leurs blasphèmes, leurs élans paniques imprègnent l'art européen d'aujourd' hui de lumière sonore, de splendeur magique, le pénètrent non seulement du mouvement de la vie charnelle, mais aussi, mais surtout de joie gratuite, de création spontanée, de fantaisie qui de ses propres inventions s'enchante et s'alimente, et enfin de cet idéo-réalisme qui le pare de l'éclat d'un printemps que l'on croyait oublié: celui de la jeunesse du monde. 